\title{
Erratum
}

27. McNutt TK, Chambers-Emerson J, Dethlefsen M, Shah R (2001) Bite the bullet: lead poisoning after ingestion of 206 lead bullets. Vet Hum Toxicol 43:288-289

28. McQuirter JL, Rothenberg SJ, Dinkins GA et al (2004) Change in blood lead concentration up to 1 year after a gunshot wound with a retained bullet. Am J Epidemiol 159:683-692

29. McQuirter JL, Rothenberg SJ, Dinkins GA et al (2001) The effects of retained lead bullets on body lead burden. J Trauma 50:892-899

30. McQuirter JL, Rothenberg SJ, Dinkins GA et al (2003) Elevated blood lead resulting from maxillofacial gunshot injuries with lead ingestion. J Oral Maxillofac Surg 61:593-603

31. Mudipalli A (2007) Lead hepatotoxicity \& potential health effects. Indian J Med Res 126:518-527

32. Nair R, Ostwal V, Sengar M et al (2011) Lead intoxication mimicking a malignancy. Hematol Oncol Stem Cell Ther 4:147-148

33. Nguyen A, Schaider JJ, Manzanares M et al (2005) Elevation of blood lead levels in emergency department patients with extra-articular retained missiles. J Trauma 58:289-299

34. Nichani V, Li WI, Smith MA et al (2006) Blood lead levels in children after phase-out of leaded gasoline in Bombay, India. Sci Total Environ 363:95-106

35. Ochsmann E, Goen T, Schaller KH, Drexler H (2009) Lead - still a health threat for marksmen. Int J Hyg Environ Health 212:557-561

36. Roszell LE, Hahn FF, Lee RB, Parkhurst MA (2009) Assessing the renal toxicity of Capstone depleted uranium oxides and other uranium compounds. Health Phys 96:343-351

37. Rusyniak DE, Arroyo A, Acciani J et al (2010) Heavy metal poisoning: management of intoxication and antidotes. Exs 100:365-396

38. Sabouraud S, Coppere B, Rousseau C et al (2009) Environmental lead poisoning from lead-glazed earthenware used for storing drinks. Rev Med Interne 30:1038-1043

39. Sabouraud S, Testud F, Descotes J et al (2008) Lead poisoning following ingestion of pieces of lead roofing plates: pica-like behavior in an adult. Clin Toxicol 46:267-269

40. Scuderi GJ, Vaccaro AR, Fitzhenry LN et al (2004) Long-term clinical manifestations of retained bullet fragments within the intervertebral disk space. J Spinal Disord Tech 17:108-111

41. Selden Al, Bergstrom BE, Gunnarsson LG (2008) Lead exposure from tourist earthenware: a pilot survey. Int J Hyg Environ Health 211:587-590

42. Verheij J, Voortman J, Nieuwkerk CM van et al (2009) Hepatic morphopathologic findings of lead poisoning in a drug addict: a case report. J Gastrointestin Liver Dis 18:225-227

43. See C von, Stuehmer A, Gellrich NC et al (2009) Wound ballistics of injuries caused by handguns with different types of projectiles. Mil Med 174:757-761

44. Willi RF, Felgenhauer N, Eyer F et al (2009) Lead intoxication in a group of workers in Germany. Dtsch Med Wochenschr 134:2556-2560

45. Wills BK, Christensen J, Mazzoncini J, Miller M (2010) Severe neurotoxicity following ingestion of tetraethyl lead. J Med Toxicol 6:31-34
Chirurg 2014 $\cdot 85: 615$

DOI 10.1007/s00104-014-2811-1

Online publiziert: 10. Juli 2014

(c) Springer-Verlag Berlin Heidelberg 2014

J. Buhr ${ }^{1}$ - E.H. Allemeyer ${ }^{1}$ K.M. Müller ${ }^{2}$ M. Glados ${ }^{3}$ M.W. Hoffmann ${ }^{1}$

${ }^{1}$ Klinik für Allgemein- und Viszeralchirurgie, Raphaelsklinik Münster

${ }^{2}$ Gerhard-Domagk-Institut für Pathologie, Universitätsklinikum Münster

${ }^{3}$ Onkologische Gemeinschaftspraxis Coesfeld

\section{Erratum zu: Polyzyklische Raumforderung im linken Gallengang}

In der html-Version des Beitrags liegt leider ein Rechtschreibfehler vor. Wir bitten, den korrekten Titel zu berücksichtigen und den Fehler zu entschuldigen. Ebenfalls ist in der html-Version die Abbildungsunterschrift von $\bullet$ Abb. 1 und 2 nicht vollständig. Wir bitten, die vollständige Bildunterschrift $\mathrm{zu}$ beachten.

\section{Korrespondenzadresse}

\section{Dr. J. Buhr}

Klinik für Allgemein- und Viszeralchirurgie, Raphaelsklinik Münster, Loerstr. 23, 48143 Münster j.buhr@raphaelsklinik.de

Die Online-Version des Originalartikels können Sie unter http://dx.doi.org/10.1007/ s00104-014-2763-5 finden.

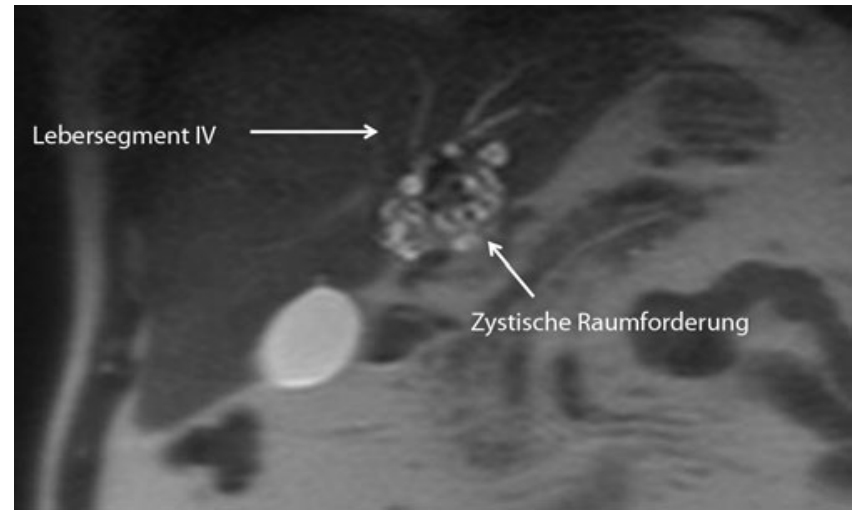

Abb. $1<$ Präoperative MRCP der polyzyklischen Raumforderung in Lebersegment III und IV. (Mit freundlicher Genehmigung von Dr. J. Weber, Radiologische Klinik, St. Vincenz-Hospital Coesfeld)

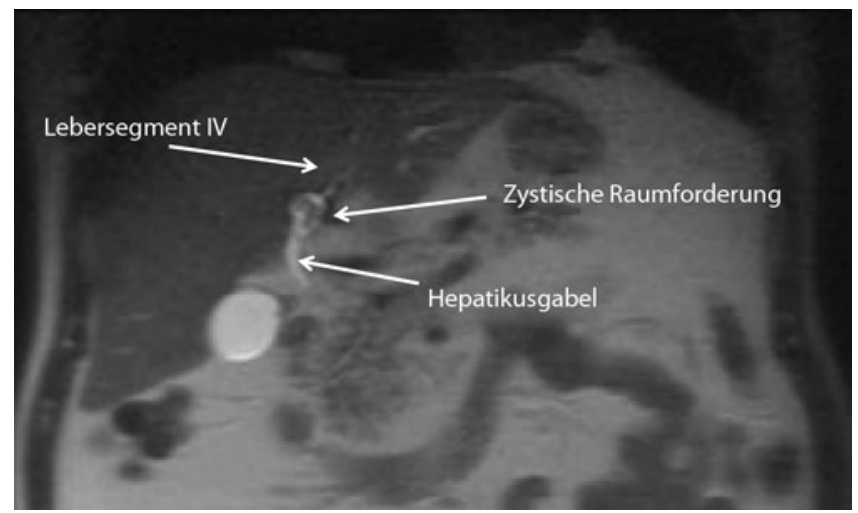

Abb. $2<$ Präoperative MRCP der polyzyklischen Raumforderung in Lebersegment III und IV mit Darstellung der Hepatikusgabel. (Mit freundlicher Genehmigung von Dr. J. Weber, Radiologische Klinik, St. Vincenz-Hospital Coesfeld) 